\title{
Disrupted dynamic network reconfiguration of the executive and reward networks in internet gaming disorder: Why IGD subjects cannot control their gaming cravings
}

\author{
Min Wang a,b,c 1, Hui Zheng d 1, Weiran Zhou a, Bo Yang a, Lingxiao Wang a,b, Shuaiyu \\ Chen a,b, Guang-Heng Dong a,b, d *
}

a Center for Cognition and Brain Disorders, School of Clinical Medicine, Hangzhou Normal University, Hangzhou, Zhejiang Province, PR China

$b$ The Affiliated Hospital of Hangzhou Normal University, Hangzhou, Zhejiang Province, PR China

c Department of Psychology, School of Humanities and Social Science, University of Science and Technology of China, Hefei, PR China

d Shanghai Jiao Tong Univ, Shanghai Mental Hlth Ctr, Sch Med, Shanghai Key Lab Psychot Disorders, Shanghai, PR China

e Zhejiang Key Laboratory for Research in Assessment of Cognitive Impairments,

Hangzhou, Zhejiang Province, PR China

1 Contributed equally.

\section{Corresponding Authors:}

Guang-Heng Dong, Ph.D., Professor

Center for Cognition and Brain Disorders, School of Clinical Medicine, Hangzhou Normal University, 2318 Yuhangtang Road, Hangzhou, Zhejiang Province, PR China. Tel.: +86 15867949909.

E-Mail: dongguangheng@hznu.edu.cn 


\begin{abstract}
Background: Studies have shown that people with internet gaming disorder (IGD) show impaired executive control over their gaming cravings; however, the neural mechanisms underlying this process remain unknown. In addition, these conclusions were based on the hypothesis that brain networks were temporally stationary and neglected changes in cognitive processes.
\end{abstract}

Methods: Resting-state fMRI data were collected from 402 subjects (162 subjects with IGD and 240 recreational game users (RGUs)). The community structure (recruitment and integration) of the executive control network and the basal ganglia network (BGN, representing the reward network) of patients with IGD and healthy controls were analyzed and compared. Mediation effects were analyzed among the different networks. Results: When compared to RGUs, subjects with IGD had a lower recruitment coefficient within the right executive control network (ECN). Further analysis showed that only male subjects had a lower recruitment coefficient. Mediation analysis showed that the integration coefficient of the right ECN mediated the relationship between the recruitment coefficients of both the right ECN and the BGN in RGUs.

Conclusions: Subjects with IGD had a lower recruitment coefficient than RGUs, and this feature was only observed in male subjects, making them less efficient at impulse control. The meditation results suggest a top-down control mechanism of the ECN is missing in subjects with IGD. All of these findings could explain why subjects with IGD have impaired executive control over their gaming cravings.

Keywords: Internet gaming disorder; community structure; executive control; recruitment 


\section{Introduction}

Many studies have proven that people with internet gaming disorder (IGD) have impaired executive control over their gaming cravings ${ }^{1-3}$. First, studies using different paradigms have shown impaired brain activation in executive control-related brain regions ${ }^{4}$, including the dorsolateral prefrontal cortex (DLPFC) and the orbitofrontal cortex $^{5,6}$. At the same time, subjects with IGD had increased gaming cravings, which could be detected by increased brain activity in reward processing-related brain regions 7-9. Second, abnormal functional connectivity (FC) between brain regions involved in executive control and reward processing has been reported in subjects with IGD 10,11. People with IGD had lower FC between the orbitofrontal cortex and the dorsal striatum 12,13 , the left medial orbitofrontal cortex and the putamen, and the DLPFC and the putamen ${ }^{14,15}$. A study with a large sample size (337 subjects) observed that subjects with IGD had lower FC between the middle frontal gyrus and the putamen, inferior frontal gyrus and ventral striatum. Disorder severity and craving scores were negatively correlated with FC between striatal and frontal brain regions ${ }^{16}$.

According to an increasing number of studies, cognitive function is accomplished by the interactions of a brain network rather than by specific regions ${ }^{17,18}$. Current studies suggest that there are two distinct brain networks that jointly influence our choices: the executive control network, which is associated with inhibiting impulses, and the reward network, which mediates immediate rewards ${ }^{19,20}$. Abnormal interactions between these two networks were observed in drug-addicted groups ${ }^{20}$, heroin-dependent subjects ${ }^{21}$, and subject with IGD ${ }^{10}$, which suggests that impairments in executive control led to ineffective inhibition of enhanced cravings for excessive online game playing. This can shed light on the mechanistic understanding of addictive behaviors at the large-scale system level. The combination of enhanced seeking motivations and an inability to inhibit impulsive behaviors is thought to represent a failure of executive control 22,23 .

Limitations existed in these studies. First, all studies described the current features of patients with IGD having impaired executive control over cravings; however, none of them could explain why subjects with IGD have disturbed processes during this process, and the neural mechanisms underlying this feature remain unclear $2,24,25$. Second, these 
researchers hypothesized that the interactive connection between brain regions/networks throughout the process was temporally stationary ${ }^{26-28}$. However, the human brain is a complex system; the active states and functional connectivity of the human brain can change during learning, growth, and even rest ${ }^{29,30}$. The brain dynamically integrates and coordinates the interaction of different brain areas to complete complex cognitive functions.

Thus, to fully understand the neural foundations of impaired executive control over gaming cravings in subjects with IGD, it is necessary to explore the dynamic characteristics of the time-dependent changes in the executive control and reward networks. In fMRI scans, the blood oxygen level dependence could signal fluctuations in brain activity; thus, there is sufficient information to study the dynamic properties of brain networks ${ }^{31}$. Several researchers have studied the variability of brain networks to detect dynamic functional changes, and they have demonstrated that it is a useful method for detecting dynamic changes in brain networks ${ }^{32}$.

Community structure is a functionally relevant graph metric to study the organization and interaction of functional systems in the brain network ${ }^{33}$. The interactive couplings within community nodes (or brain regions) are strong and dense, whereas interactive couplings between communities are sparse ${ }^{34}$. The community structure (or modular organization) can delineate the functional segregation and integration of whole-brain networks. In most cases, two indices were used to measure the community features: recruitment, which refers to the probability that a brain region is in the same community as other nodes from its own network; and integration, which refers to the probability that a brain region is in the same community as nodes from other networks 35. Researchers have identified community structure in both structural and functional networks in the healthy human brain ${ }^{36}$. Community structure has been used to identify potential functional modules for groups of brain regions exhibiting similar trajectories and functions over time 36,37 . The way networks change over time can provide insights into neural mechanisms.

Recently, using community structure has piqued the interest of clinical researchers, as studies have proven that disrupted community structures are found in a variety of brain 
disorders ${ }^{38,39}$. A study found a less stable community structure at the resting-state network level in a group of patients with schizophrenia and provided novel methods for exploring dynamic community structure ${ }^{40}$. Lord et al. detected the community structure of the functional network for individuals with unipolar depression ${ }^{41}$. Zheng et al. showed that, in major depression disorder (MDD), the anterior cingulate cortex exhibited abnormal flexibility in community structures ${ }^{42}$ and that unmedicated MDD groups and medicated MDD groups exhibited a similar reconfiguration of the community structure of the visual association and the default mode systems, but that the groups had different reconfiguration profiles in the frontoparietal control subsystems ${ }^{43}$. One study suggested that dynamic network measures may be an effective biomarker for detecting language dysfunction associated with neurological diseases like temporal lobe epilepsy $^{35}$.

The primary aim of the current study was to address the limitations of previous studies on IGD and provide a better understanding of the neural mechanisms underlying impaired executive control over cravings. To achieve this goal, we examined the distribution of community assignment across the entire scanning time and compared the changes in the community structure of the executive control network and reward network in subjects with IGD. We hypothesized that patients with IGD would have a different community structure than the healthy control group. Second, we wanted to explore how these community structure features affect the top-down controlling process and whether this feature can be observed in subjects with IGD. We hypothesized that the top-down control process is disrupted in subjects with IGD.

\section{Methods and procedures}

\section{Participants}

This study was approved by the ethics investigation committee of Hangzhou Normal University and conducted from Dec 2013 to Dec 2019. All participants signed written informed consent forms in accordance with the Declaration of Helsinki.

Four hundred thirty (430) online game players were recruited from universities in China. Twelve players with missing fMRI data and sixteen players with head motions > $2.5^{\circ}$ during the scan were excluded. The remaining subjects were evaluated for the severity of IGD via both Young's Internet Addiction Test (IAT) (www.netaddiction.net) 
and DSM-5 diagnostic criteria ${ }^{44}$. Individuals with IGD had to have IAT scores greater than 50, as well as at least five of the nine DSM-5 criteria (as they were recruited before the release of DSM-5, 41 subjects from Dec 2013 did not have DSM-5 data). A total of 162 subjects were diagnosed with IGD, and the remaining 240 were classified as recreational game users (RGUs) according to the assessment by trained researchers. For relevant demographic information, see Table 1. All subjects had no comorbid DSM-IV axis I or axis II disorders, based on the results of a structured psychiatric interview ${ }^{45}$.

\section{-Insert Table 1 here}

\section{Imaging acquisition}

Resting-state functional data were acquired from a 3T Siemens Trio MRI system using an echo-planar imaging (EPI) pulse sequence(33 axial slices with $3 \mathrm{~mm}$ slice thickness, $\mathrm{TR}=2000 \mathrm{~ms}, \mathrm{TE}=30 \mathrm{~ms}$, matrix $=64 \times 64, \mathrm{FOV}=220 \times 220 \mathrm{~mm}$, flip angle $=$ $90^{\circ}$, and a total of 210 volumes). The structural images were from a high-resolution, T1weighted magnetization-prepared rapid gradient echo sequence $(\mathrm{TR}=1900 \mathrm{~ms}, \mathrm{TE}=$ $2.52 \mathrm{~ms}, \mathrm{TI}=900 \mathrm{~ms}$, flip angle $=9^{\circ}$, matrix $=256 \times 256$, slices $=176$, voxel size $=1 \times 1$ $\times 1 \mathrm{~mm}^{3}$ ).

\section{Image preprocessing}

The DPABI version 5.2 toolbox was used to perform fMRI preprocessing ${ }^{46}$. The first 10 volumes were removed for all data. Slice-time and motion parameters were then evaluated and corrected for the remaining volumes. For the data in individual space, spatial normalization with the EPI templates was carried out and transformed to the MNI space with a $2 \times 2 \times 2 \mathrm{~mm}^{3}$ voxel resolution. Normalized data were smoothed with a 6 FWHM Gaussian kernel. Finally, the obtained images were detrended and regressed as covariates with noisy signals from white matter (WM), cerebrospinal fluid (CSF) and head motion parameters (Friston 24 model).

\section{Network ROI selection}

IGD is often accompanied by dysfunctions in frontostriatal circuits, which can be interpreted as a disconnection between the executive control network and the basal ganglia network. We used the Shirer et al. ${ }^{47}$ atlas, which covers 14 resting-state 
networks, to define these functional networks a priori, and the left and right executive control networks (including 6 brain regions per network) and the basal ganglia network (5 regions) were extracted for the current analysis. The right executive control network and the basal ganglia network overlap in the right caudate nucleus and the middle frontal gyrus due to the functional diversity of the regions. The time correlation $r$ value between each voxel within the overlapping area and the two networks was calculated using all subject data, and the right caudate and middle frontal gyrus were parcellated by using a "winner-takes-all" approach with a bootstrapping strategy ${ }^{48}$ (see Fig. 1).

\section{Network construction}

We extracted the mean time series of 17 functionally defined regions of interest (ROIs). For network construction, we used wavelet decomposition rather than functional connectivity to extract information from these time series because it is more sensitive to subtle signal changes in nonstationary time series with noisy backgrounds 49. We use a maximal overlap discrete wavelet transformation with the Daubechies least asymmetric approach to decompose the time series into multiple frequency intervals 50 : scale 1, 0.125 0.25 Hz; scale 2, 0.062 0.125 Hz; scale 3, 0.031 0.062 Hz; and scale 4, $0.015 \sim 0.031 \mathrm{~Hz}$. Scale 3 was chosen as the main analysis frequency band because it is completely covered by the frequency range commonly interpreted in resting fMRI (i.e., $0.01 \sim 0.1$ ) and has higher sensitivity to disease classification ${ }^{51}$.

The time-series data were then split into a consecutive series of $40 \mathrm{~s}$ time windows that overlapped with contiguous windows by 50\%. The magnitude-squared spectral coherence between each pair of ROIs was estimated according to ${ }^{52,53}$, generating a $17 \times$ 17 adjacency matrix for each time window. Finally, the adjacency matrices in all 19 time windows would be linked together to form a multilayer network.

\section{Multilayer community detection}

A community describes a group of nodes that are more strongly connected to each other than to nodes outside of their community ${ }^{54}$, whereas a multilayer community further characterizes their reconfiguration over time. In the current study, we used a 
generalized Louvain community detection algorithm ${ }^{33}$ involving the following multilayer modularity quality function:

$$
\mathbf{Q}=\frac{1}{2 \mu} \sum_{i j l r}\left[\left(\mathbf{A}_{i j l}-\gamma_{l} \mathbf{V}_{i j l}\right) \boldsymbol{\delta}_{l r}+\delta_{i j} \omega_{j l r}\right] \delta\left(\mathbf{g}_{i l}, \mathbf{g}_{j r}\right)
$$

where $\mu$ is the total edge weight of the network, $A_{i j l}$ is the edge between nodes $i$ and $j$ at layer $l$ of the multilayer network, and $V_{i j l}$ describes the corresponding element of a null model. The parameter $\gamma_{l}$ sets the structural resolution parameter of layer $l$ (i.e., the weight of intralayer edges) the parameter $\omega_{j l r}$ sets the temporal resolution parameter (i.e., the weight of interlayer edges, here $\gamma_{l}=1, \omega_{j l r}=0.4$ ) ${ }^{55}$, and the parameter $g$ describes the community assignments of two nodes across the time domain, involving node $i$ in layer $l$ and node $j$ in layer $r . \delta$ is a Kronecker delta function, where $\delta(g i l, g j r)=1$ if $i l=j r$ and 0 otherwise.

Although the current network should be considered orderly and have interlayer links between sequential layers for nodes at the same position, the generalized Louvain algorithm has a stochastic nature, sometimes causing the instability of community assignments ${ }^{56}$. To ensure the stability of the results, we performed 100 iterations for each subject and calculated the mean, similar to an implementation used in previous studies ${ }^{35}$.

\section{Recruitment and integration}

The 17 ROIs were categorized into three resting-state networks: the left and right executive control networks and the basal ganglia network (reward network). We calculated two dynamic indicators to quantify the dynamic interactions on inter- or intranetworks: recruitment and integration. The recruitment coefficient describes the average probability that node $i$ is in the same community as other nodes from its own network and is defined as:

$$
\mathbf{R}_{i}^{N}=\frac{1}{m_{N}} \sum_{j \in N} \mathbf{P}_{i j}
$$

where $m_{N}$ is the size of network $N$, calculated as the number of nodes in $N$ and $P_{i j}$ corresponds to the relative frequency at which nodes $i$ and $j$ were assigned to the same 
community across the time domain, where $P i j=1$ if nodes $i$ and $j$ are always in the same community and 0 otherwise. Therefore, a node with high recruitment tends to be associated with nodes from its own network in the time domain. The integration coefficient describes the average probability that node $i$ is in the same community as nodes from other networks, given by:

$$
\mathbf{I}_{i}^{N}=\frac{1}{K-m_{N}} \sum_{j \notin N} \mathbf{P}_{i j}
$$

where $K$ is the total number of nodes. A node with high integration tends to be associated with nodes from other networks in the time domain.

\section{Statistical analysis}

Statistical analyses were conducted using SPSS 20.0. To match the two groups of subjects, we chose subjects from high to low according to their IAT scores. The first third of the subjects (134 subjects per group) were included. All statistical processes were performed at the network level, and significance was determined using independent sample t-tests with Bonferroni correction $(p<.016)$. For the exploratory mediation analysis, PROCESS bootstrapping and bias-corrected 95\% confidence intervals were used to assess the significance of the mediation model ${ }^{57}$; a CI that does not contain 0 indicates a significant mediation effect. The analysis pipeline is depicted in Fig. 2.

\section{Results}

\section{Recruitment and integration}

Compared to RGUs, subjects with IGD had a lower recruitment coefficient within RECNs $(t=-2.689, p=0.007$, Bonferroni correction, see Fig. 3A). In general, a lower recruitment coefficient indicates that the nodes within the network are less likely to be divided into the same community over time. Thus, the current results might suggest that the functional network characterized by executive control in subjects with IGD was decoupled from the dynamic process. Although our hypothesis was associated with altered dynamic interactions between the ECN and BGN in subjects with IGD, no 
significant differences in the integration coefficient were found between the groups (Fig. 3B).

In addition, we looked at the differences by sex in recruitment coefficients within RECNs. The results showed that there was a significant difference in the RECN recruitment coefficient only among males $(t=-2.467, p=0.015)$, Bonferroni correction, see Fig. 4.

\section{Mediation analysis}

To further verify our hypothesis, an exploratory analysis of differences in the RCEN between groups was conducted by a mediation model. First, in the RGUs, we observed a two-way correlation of the integration coefficient of the RECN with both the recruitment coefficient of the RECN $(r=-0.225, p=0.009)$ and of the BGN $(r=0.276, p=$ 0.001), whereas no similar correlations were found in subjects with IGD. We then performed a mediation analysis using the integration coefficient of the RECN as the mediating factor, which showed that the integration coefficient of the RECN mediates the relationship between the recruitment coefficients of the RECN and BGN in RGUs (see Fig. 5B). The same model did not hold for subjects with IGD.

Insert Fig. 5 here

\section{Statistical null models}

We adopted the network null model to quantify the dynamic modular organization of the resting state network in subjects with IGD and RGUs ${ }^{52,58}$. Descriptions and comparison results of these null models are provided in the Supplementary material. In short, the current results supported the dynamics of real network modules.

\section{Replication test with longitudinal data}

To investigate further, we tracked 40 subjects (22 IGD, 18 RGU) (these 40 subjects were part of the 402 subjects) for more than 6 months and obtained additional data. We 
collected their resting-state data and addiction features during these two times of scanning. All the data analyses steps were of the same to those in the cross-sectional analyses. We compared the post-pre test and observed a decrease of the recruitment in network community in those 22 IGD subjects $(t=-1.467, p=0.095)$, although the change did not reach the statistical significance. This suggests that continuous IGD situation further impaired their recruitment feature in executive control network. The result provides additional support to the conclusions.

\section{Discussion}

Using the dynamic network analysis method, the current study provides a new perspective on the dynamic reconfiguration of the executive control network and reward network in subjects with IGD. The current study found a disturbed community structure (recruitment) in subjects with IGD, which may explain why subjects with IGD have impaired executive control abilities. Further analyses showed that the decreased recruitment coefficient was only observed in male subjects. This provides further support for previous findings on sex differences in IGD. We also explored the mediation effect between different networks.

\section{Subjects with IGD show disturbed community structure in the executive control network}

'Recruitment' is the probability that a brain region is assigned to the same community as other nodes from its own network, and it is used to quantify the probability that a functionally-defined region of interest is assigned to the same community as functionally-defined regions of interest from the same subsystem ${ }^{17,18}$. Generally, a reduced recruitment coefficient indicates that the nodes within the network are less likely to be divided into the same community over time.

In the current study, we learned from healthy controls that during the scanning period, the executive control process recruited stable regions within the executive control network and the reward network. Regions within these two systems exhibited a higher preference for intra-subsystem communication but a relatively low preference for intersubsystem communication. However, in subjects with IGD, recruitment in the executive control network was unstable, and regions within the executive control network 
exhibited a lower preference for intra-subsystem communication but a relatively high preference for inter-subsystem communication. This suggests that subjects with IGD have an unstable executive network, and that the brain regions they recruit may differ each time they perform executive control. This disturbs the efficiency of executive control and prevents subjects with IGD from effectively controlling their impulses. For the reward network, there was no difference between the two groups.

Based on the patterns of recruitment features we observed, we concluded that subjects with IGD have a disturbed community structure in the executive control network; they had a lower preference for intra-subsystem communication but a relatively high preference for inter-subsystem communication. The decreased recruitment feature disrupted the efficiency of their executive control, which could explain why subjects with IGD have disrupted executive control and find it harder to control their gaming cravings.

\section{Disturbed community structure in male but not female subjects with IGD}

Further analysis showed that the differences in recruitment between groups was only observed in male subjects with IGD, and no such features were observed in female subjects with IGD. As discussed in the introduction, studies have observed impaired executive control over reward seeking and craving for gaming ${ }^{1-3}$; however, sex differences were observed in IGD studies. First, the prevalence of IGD is higher in males than in females, which suggests that males are more likely than females to develop IGD 59. At the same time, male subjects with IGD show more impaired executive control than female subjects with IGD, and it is harder for males to control their gaming cravings. The inhibitory control over game-elicited cravings in male subjects with IGD ' was more severely disrupted by gaming cues than that of females ${ }^{60}$; short-term gaming elicited more craving-related activations to gaming cues in males vs. females ${ }^{61}$. Brain regions implicated in executive control showed differential functional connectivity in males during gaming ${ }^{62}$. Even for recreational gamers, female players exhibit better executive control than male players when facing gaming cues, which may provide resilience against developing IGD ${ }^{7}$. 
In the current study, decreased recruitment was only observed in male subjects with IGD, not females, and these findings provide further support to studies on sex differences in IGD. As we discussed on the role of recruitment, we can conclude that male subjects with IGD are less efficient in recruiting their executive control network regions, which provides an explanation for the underpinnings of the sex differences in executive control in IGD.

\section{The meditation effects suggest that subjects with IGD lack top-down control}

\section{function}

We also observed in RGUs that the integration coefficient of the RECN mediates the relationship between the recruitment of both the RECN and the BGN, which was not observed in subjects with IGD. The integration coefficient of the RECN reflected the dynamic interaction between nodes in the RECN and other networks. According to the framework of addictions, addiction is the result of an imbalance between the ECN and the BGN, which are generally considered to be structurally independent but functionally interacting ${ }^{63}$. After an individual learns social rules, the ECN, which is primarily located in the frontal lobe, controls the subcortical BGN through several mechanisms, including decision-making and inhibitory control, which is considered to be the top-down control mechanism of the ECN in addiction ${ }^{64,65}$. Under current conditions, we speculated that there was a stable top-down regulation mechanism based on a specific mediation framework in RGUs. However, this appears to be missing in subjects with IGD, which might be a potential risk factor for why IGD tends to be associated with dysfunctions of executive control.

\section{Limitations}

Several limitations should be addressed. First, all subjects in the current study were free of comorbid disorders to exclude potential extra effects from other disorders. However, IGD is usually comorbid with other disorders, such as ADHD, nicotine addiction, or other types of psychiatric disorders. Second, all data were cross-sectional and lacked longitudinal data to verify the causal relationship between IGD and impaired executive control. Future studies should investigate this issue. 


\section{Conclusions}

First, subjects with IGD showed a lower recruitment coefficient than RGUs, making them less efficient at controlling their impulses. This could explain why subjects with IGD have impaired executive control over their gaming cravings. Second, decreased recruitment was observed only in male subjects with IGD, which is consistent with previous studies on sex differences in IGD. Third, the top-down regulation mechanism was only observed in RGUs but not in subjects with IGD, which suggests that subjects with IGD lack a stable top-down regulation mechanism. These conclusions revealed the mechanisms underlying why subjects with IGD exhibit impaired executive control over gaming cravings.

\section{Statistics and reproducibility}

All statistical steps were used open software and we did not do any modification on them. The parameters were provided on each of the statistical steps.

\section{Acknowledgements}

The current research was supported by The Cultivation Project of Province-levelled Preponderant Characteristic Discipline of Hangzhou Normal University (20JYXK008) and the Zhejiang Provincial Natural Science Foundation (LY20C090005).

This article has been posted on the preprint server BioRxiv.

\section{Author contributions}

Min Wang designed this research and wrote the first draft of the manuscript. Min Wang and Hui Zheng analyzed the data and prepared the figures and tables and the longitudinal data analyses; Weiran Zhou, Lingxiao Wang, Shuaiyu Chen contributed to fMRI data collection, and manuscript revision. Guang-Heng Dong designed this research and edited the manuscript. All authors contributed to and approved the final manuscript.

\section{Conflicts of interest}

The authors declare no competing interests. 


\section{Data Availability}

The data stored at our lab based network attachment system:

http://QuickConnect.cn/others. ID:guests; PIN dong@123.COM

\section{References}

1 Wang, M., Dong, H., Zheng, H., Du, X. \& Dong, G. H. Inhibitory neuromodulation of the putamen to the prefrontal cortex in Internet gaming disorder: How addiction impairs executive control. J Behav Addict 9, 312-324, doi:10.1556/2006.2020.00029 (2020).

2 Weinstein, A. \& Lejoyeux, M. Neurobiological mechanisms underlying internet gaming disorder. Dialogues Clin Neurosci 22, 113-126, doi:10.31887/DCNS.2020.22.2/aweinstein (2020).

3 Dong, G. \& Potenza, M. N. A cognitive-behavioral model of Internet gaming disorder: theoretical underpinnings and clinical implications. Journal of psychiatric research 58, 7-11, doi:10.1016/j.jpsychires.2014.07.005 (2014).

4 Dong, G., Li, H., Wang, L. \& Potenza, M. N. Cognitive control and reward/loss processing in Internet gaming disorder: Results from a comparison with recreational Internet game-users. European psychiatry : the journal of the Association of European Psychiatrists 44, 30-38, doi:10.1016/j.eurpsy.2017.03.004 (2017).

5 Brand, M., Young, K. S. \& Laier, C. Prefrontal control and internet addiction: a theoretical model and review of neuropsychological and neuroimaging findings. Frontiers in human neuroscience 8, 375, doi:10.3389/fnhum.2014.00375 (2014).

6 Zheng, H. et al. Meta-analyses of the functional neural alterations in subjects with Internet gaming disorder: Similarities and differences across different paradigms. Progress in neuro-psychopharmacology \& biological psychiatry 94, 109656, doi:10.1016/j.pnpbp.2019.109656 (2019).

7 Dong, G. et al. Gender-related differences in cue-elicited cravings in Internet gaming disorder: The effects of deprivation. J Behav Addict 7, 953-964, doi:10.1556/2006.7.2018.118 (2018).

8 Chun, J. W. et al. Altered core networks of brain connectivity and personality traits in internet gaming disorder. J Behav Addict 9, 298-311, doi:10.1556/2006.2020.00014 (2020).

9 Ma, S. S. et al. Alterations in functional networks during cue-reactivity in Internet gaming disorder. J Behav Addict 8, 277-287, doi:10.1556/2006.8.2019.25 (2019).

10 Dong, G., Lin, X., Hu, Y., Xie, C. \& Du, X. Imbalanced functional link between executive control network and reward network explain the online-game seeking behaviors in Internet gaming disorder. Scientific reports 5, 9197, doi:10.1038/srep09197 (2015). 
11 Wang, M. et al. Altered effective connectivity from the pregenual anterior cingulate cortex to the laterobasal amygdala mediates the relationship between internet gaming disorder and loneliness. Psychol Med, 1-10, doi:10.1017/S0033291720002366 (2020).

12 Wang, Y. et al. Impaired executive control and reward circuit in Internet gaming addicts under a delay discounting task: independent component analysis. European archives of psychiatry and clinical neuroscience 267, 245-255, doi:10.1007/s00406016-0721-6 (2017).

13 Dong, G. H. et al. Dorsal and ventral striatal functional connectivity shifts play a potential role in internet gaming disorder. Commun Biol 4, 866, doi:10.1038/s42003021-02395-5 (2021).

14 Han, X. et al. Alterations of Resting-State Static and Dynamic Functional Connectivity of the Dorsolateral Prefrontal Cortex in Subjects with Internet Gaming Disorder. Frontiers in human neuroscience 12, 41, doi:10.3389/fnhum.2018.00041 (2018).

15 Dong, G. H. et al. Disrupted prefrontal regulation of striatum-related craving in Internet gaming disorder revealed by dynamic causal modeling: results from a cuereactivity task. Psychol Med 51, 1549-1561, doi:10.1017/S003329172000032X (2021).

16 Dong, H. et al. Reduced frontostriatal functional connectivity and associations with severity of Internet gaming disorder. Addiction biology 26, e12985, doi:10.1111/adb.12985 (2021).

17 Bassett, D. S. \& Mattar, M. G. A Network Neuroscience of Human Learning: Potential to Inform Quantitative Theories of Brain and Behavior. Trends in cognitive sciences 21, 250-264, doi:10.1016/j.tics.2017.01.010 (2017).

18 Bassett, D. S. \& Sporns, O. Network neuroscience. Nature neuroscience 20, 353-364, doi:10.1038/nn.4502 (2017).

19 McClure, S. M., Ericson, K. M., Laibson, D. I., Loewenstein, G. \& Cohen, J. D. Time discounting for primary rewards. The Journal of neuroscience : the official journal of the Society for Neuroscience 27, 5796-5804, doi:10.1523/JNEUROSCI.4246-06.2007 (2007).

20 Monterosso, J., Piray, P. \& Luo, S. Neuroeconomics and the study of addiction. Biological psychiatry 72, 107-112, doi:10.1016/j.biopsych.2012.03.012 (2012).

21 Xie, C. et al. Imbalanced functional link between valuation networks in abstinent heroin-dependent subjects. Molecular psychiatry 19, 10-12, doi:10.1038/mp.2012.169 (2014).

22 Goldstein, R. Z. \& Volkow, N. D. Drug addiction and its underlying neurobiological basis: neuroimaging evidence for the involvement of the frontal cortex. The American journal of psychiatry 159, 1642-1652, doi:10.1176/appi.ajp.159.10.1642 (2002).

23 Volkow, N. D. et al. Cognitive control of drug craving inhibits brain reward regions in cocaine abusers. Neurolmage 49, 2536-2543, doi:10.1016/j.neuroimage.2009.10.088 (2010).

24 Brand, M. et al. The Interaction of Person-Affect-Cognition-Execution (I-PACE) model for addictive behaviors: Update, generalization to addictive behaviors beyond internet-use disorders, and specification of the process character of addictive behaviors. Neurosci Biobehav Rev 104, 1-10, doi:10.1016/j.neubiorev.2019.06.032 (2019). 
25 Petry, N. M., Zajac, K. \& Ginley, M. K. Behavioral Addictions as Mental Disorders: To Be or Not To Be? Annu Rev Clin Psychol 14, 399-423, doi:10.1146/annurev-clinpsy032816-045120 (2018).

26 Damoiseaux, J. S. et al. Consistent resting-state networks across healthy subjects. Proceedings of the National Academy of Sciences of the United States of America 103, 13848-13853, doi:10.1073/pnas.0601417103 (2006).

27 Friston, K. J. Functional and effective connectivity: a review. Brain connectivity 1, 1336, doi:10.1089/brain.2011.0008 (2011).

28 Power, J. D. et al. Functional network organization of the human brain. Neuron 72, 665-678, doi:10.1016/j.neuron.2011.09.006 (2011).

29 Medaglia, J. D., Lynall, M. E. \& Bassett, D. S. Cognitive network neuroscience. Journal of cognitive neuroscience 27, 1471-1491, doi:10.1162/jocn_a_00810 (2015).

30 Supekar, K., Musen, M. \& Menon, V. Development of large-scale functional brain networks in children. PLoS Biol 7, e1000157, doi:10.1371/journal.pbio.1000157 (2009).

31 Handwerker, D. A., Roopchansingh, V., Gonzalez-Castillo, J. \& Bandettini, P. A. Periodic changes in fMRI connectivity. Neurolmage 63, 1712-1719, doi:10.1016/j.neuroimage.2012.06.078 (2012).

32 Chang, C. \& Glover, G. H. Time-frequency dynamics of resting-state brain connectivity measured with fMRI. Neurolmage 50, 81-98, doi:10.1016/j.neuroimage.2009.12.011 (2010).

33 Mucha, P. J., Richardson, T., Macon, K., Porter, M. A. \& Onnela, J. P. Community structure in time-dependent, multiscale, and multiplex networks. Science 328, 876878, doi:10.1126/science.1184819 (2010).

34 Newman, M. E. \& Girvan, M. Finding and evaluating community structure in networks. Phys Rev E Stat Nonlin Soft Matter Phys 69, 026113, doi:10.1103/PhysRevE.69.026113 (2004).

$35 \mathrm{He}, \mathrm{X}$. et al. Disrupted dynamic network reconfiguration of the language system in temporal lobe epilepsy. Brain : a journal of neurology 141, 1375-1389, doi:10.1093/brain/awy042 (2018).

36 Crossley, N. A. et al. Cognitive relevance of the community structure of the human brain functional coactivation network. Proceedings of the National Academy of Sciences of the United States of America 110, 11583-11588, doi:10.1073/pnas.1220826110 (2013).

37 Fortunato, S. Community detection in graphs. Phys Rep 486, 75-174, doi:10.1016/j.physrep.2009.11.002 (2010).

38 Lerman-Sinkoff, D. B. \& Barch, D. M. Network community structure alterations in adult schizophrenia: identification and localization of alterations. Neuroimage Clin 10, 96-106, doi:10.1016/j.nicl.2015.11.011 (2016).

39 Cary, R. P. et al. Network Structure among Brain Systems in Adult ADHD is Uniquely Modified by Stimulant Administration. Cerebral cortex 27, 3970-3979, doi:10.1093/cercor/bhw209 (2017).

40 Gifford, G. et al. Resting state fMRI based multilayer network configuration in patients with schizophrenia. Neuroimage Clin 25, 102169, doi:10.1016/j.nicl.2020.102169 (2020). 
41 Lord, A., Horn, D., Breakspear, M. \& Walter, M. Changes in Community Structure of Resting State Functional Connectivity in Unipolar Depression. Plos One 7, doi:ARTN e4128210.1371/journal.pone.0041282 (2012).

42 Zheng, H. et al. The dynamic characteristics of the anterior cingulate cortex in resting-state $\mathrm{fMRI}$ of patients with depression. Journal of affective disorders 227, 391-397, doi:10.1016/j.jad.2017.11.026 (2018).

$43 \mathrm{He}, \mathrm{Y}$. et al. Reconfiguration of Cortical Networks in MDD Uncovered by Multiscale Community Detection with fMRI. Cerebral cortex 28, 1383-1395, doi:10.1093/cercor/bhx335 (2018).

44 APA. Diagnostic and statistical manual of mental disorders: DSM-5 ${ }^{\mathrm{TM}}$ (5th ed.). (2013).

45 Sheehan, D. V. et al. The Mini-International Neuropsychiatric Interview (M.I.N.I.): the development and validation of a structured diagnostic psychiatric interview for DSMIV and ICD-10. The Journal of clinical psychiatry 59 Suppl 20, 22-33; quiz 34-57, doi:10.4088/JCP.09m05305whi (1998).

46 Yan, C.-G., Wang, X.-D., Zuo, X.-N. \& Zang, Y.-F. DPABI: Data Processing \& Analysis for (Resting-State) Brain Imaging. Neuroinformatics 14, 339-351, doi:10.1007/s12021016-9299-4 (2016).

47 Shirer, W. R., Ryali, S., Rykhlevskaia, E., Menon, V. \& Greicius, M. D. Decoding Subject-Driven Cognitive States with Whole-Brain Connectivity Patterns. Cereb. Cortex 22, 158-165, doi:10.1093/cercor/bhr099 (2012).

48 He, X. S., Doucet, G. E., Sperling, M., Sharan, A. \& Tracy, J. I. Reduced thalamocortical functional connectivity in temporal lobe epilepsy. Epilepsia 56, 1571-1579, doi:10.1111/epi.13085 (2015).

49 Brammer, M. J. Multidimensional wavelet analysis of functional magnetic resonance images. Human Brain Mapping 6, 378-382, doi:10.1002/(sici)10970193(1998)6:5/6<378::aid-hbm9>3.0.co;2-7 (1998).

50 Cazelles, B. et al. Wavelet analysis of ecological time series. Oecologia 156, 287-304, doi:10.1007/s00442-008-0993-2 (2008).

51 Wang, J. H. et al. Disrupted Functional Brain Connectome in Individuals at Risk for Alzheimer's Disease. Biological Psychiatry 73, 472-481, doi:10.1016/j.biopsych.2012.03.026 (2013).

52 Bassett, D. S. et al. Dynamic reconfiguration of human brain networks during learning. Proceedings Of the National Academy Of Sciences Of the United States Of America 108, 7641-7646, doi:10.1073/pnas.1018985108 (2011).

$53 \mathrm{He}, \mathrm{X}$. S. et al. Disrupted dynamic network reconfiguration of the language system in temporal lobe epilepsy. Brain 141, 1375-1389, doi:10.1093/brain/awy042 (2018).

54 Newman, M. E. J. Modularity and community structure in networks. Proceedings Of the National Academy Of Sciences Of the United States Of America 103, 8577-8582, doi:10.1073/pnas.0601602103 (2006).

55 Chai, L. R., Mattar, M. G., Blank, I. A., Fedorenko, E. \& Bassett, D. S. Functional Network Dynamics of the Language System. Cerebral Cortex 26, 4148-4159, doi:10.1093/cercor/bhw238 (2016).

56 Good, B. H., de Montjoye, Y. A. \& Clauset, A. Performance of modularity maximization in practical contexts. Physical Review E 81, doi:10.1103/PhysRevE.81.046106 (2010). 
57 Hayes, A. F. PROCESS : A Versatile Computational Tool for Mediation, Moderation, and Conditional Process Analysis. (2012).

58 Bassett, D. S. et al. Robust detection of dynamic community structure in networks. Chaos 23, doi:10.1063/1.4790830 (2013).

59 Pan, Y. C., Chiu, Y. C. \& Lin, Y. H. Systematic review and meta-analysis of epidemiology of internet addiction. Neurosci Biobehav Rev 118, 612-622, doi:10.1016/j.neubiorev.2020.08.013 (2020).

60 Zhou, W. et al. Sex difference in neural responses to gaming cues in Internet gaming disorder: Implications for why males are more vulnerable to cue-induced cravings than females. Neuroscience letters 760, 136001, doi:10.1016/j.neulet.2021.136001 (2021).

61 Dong, G., Wang, L., Du, X. \& Potenza, M. N. Gender-related differences in neural responses to gaming cues before and after gaming: implications for gender-specific vulnerabilities to Internet gaming disorder. Social cognitive and affective neuroscience 13, 1203-1214, doi:10.1093/scan/nsy084 (2018).

62 Dong, G., Wang, Z., Wang, Y., Du, X. \& Potenza, M. N. Gender-related functional connectivity and craving during gaming and immediate abstinence during a mandatory break: Implications for development and progression of internet gaming disorder. Progress in neuro-psychopharmacology \& biological psychiatry 88, 1-10, doi:10.1016/j.pnpbp.2018.04.009 (2019).

63 Noel, X., Brevers, D. \& Bechara, A. A neurocognitive approach to understanding the neurobiology of addiction. Current Opinion In Neurobiology 23, 632-638, doi:10.1016/j.conb.2013.01.018 (2013).

64 Bechara, A. Decision making, impulse control and loss of willpower to resist drugs: a neurocognitive perspective. Nat. Neurosci. 8, 1458-1463, doi:10.1038/nn1584 (2005).

65 Bechara, A. et al. A Neurobehavioral Approach to Addiction: Implications for the Opioid Epidemic and the Psychology of Addiction. Psychological Science In the Public Interest 20, 96-127, doi:10.1177/1529100619860513 (2019). 


\section{Figure Legends}

\section{Fig.1 Segmentation of right caudate and middle frontal gyrus}

These areas are divided into two networks based on the "winner-take-all" strategy. For the caudate, the area marked in pink is assigned as RECN, and the area marked in purple is assigned as BGN. For the middle frontal gyrus, the area marked in blue is assigned as RECN, and the area marked in purple is assigned as BGN.

\section{Fig. 2 The analytic pipeline in current study}

This figure shows the whole process from pre-processing to statistical analysis.

\section{Fig.3 Between-group differences in recruitment and integration coefficients}

Plot.A: left plot shows between-group difference in recruitment coefficient and right plot is a schematic diagram of the recruitment coefficient in a dynamic community; Plot.B: left plot shows between-group difference in integration coefficient and right plot is a schematic diagram of the integration coefficient in a dynamic community. ${ }^{* *}: p<$ 0.01 .

\section{Fig.4 Between-group differences in recruitment coefficients based on sex}

Left plot shows between-group difference in recruitment coefficient for males and females, while right plot is a schematic diagram of the recruitment coefficient in a dynamic community. *: $p<0.05$.

\section{Fig.5 Mediation analysis}

Left plot shows correlations of integration in RECN with the recruitment in BGN and RECN in RGUs, while right plot shows that integration in RECN significantly mediate the relationships between recruitment in BGN and RECN in RGUs. 


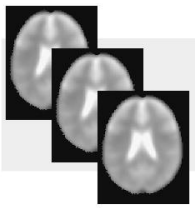

fMRI preprocessing
ROI 01

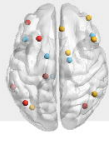

6

ROI 02
Network's ROI selection

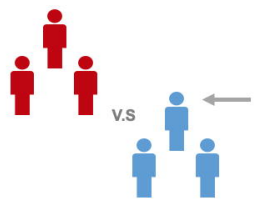

Community 1

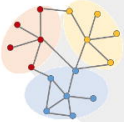

Community 2

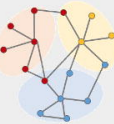

Time
Community 19
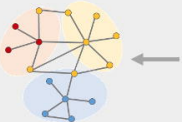

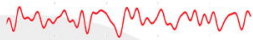



\section{Wavelet decompose}
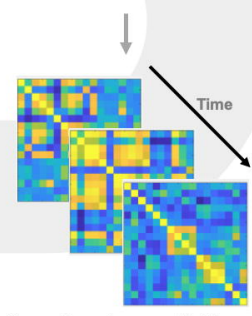

Dynamic coherence Matrices 
(A)

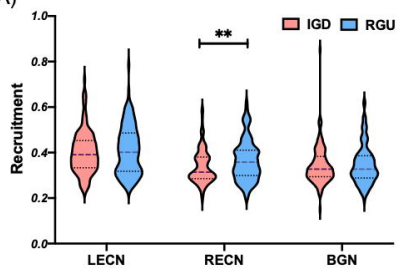

Community 1

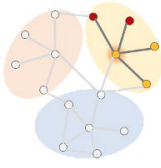

Community 19

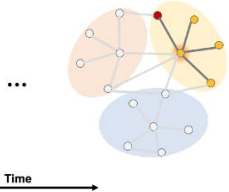

Time
Community 1

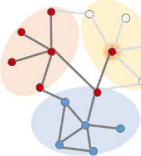

Community 19



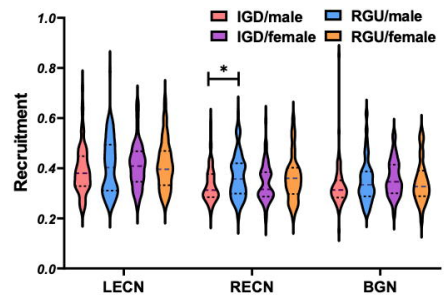

Community 1

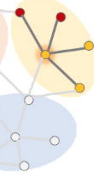

Community 19

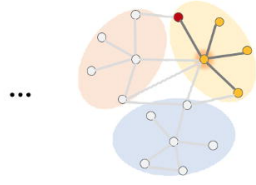


(A)

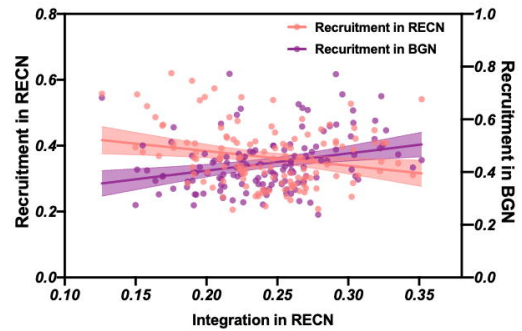

(B)



